\title{
The role of the Infectious Diseases Unit at Groote Schuur Hospital in addressing South Africa's greatest burden of disease
}

\author{
Mishal Pandie, Helen van der Plas, Gary Maartens, Marc Mendelson
}

Background. The greatest burden of disease in South Africa (SA) comes from infectious diseases (ID), with human immunodeficiency virus (HIV) and tuberculosis (TB) dominating the health landscape. However, other infections including community-acquired and imported infections and the rise in hospital-acquired infections pose a considerable threat to public health.

Methods and objectives. We used a prospective cross-sectional study to examine the profile of patients referred to the Infectious Diseases Unit at Groote Schuur Hospital (GSH) between 2008 and 2011.

Results. A total of 2142 patient consultations were performed, the majority at the request of secondary hospital level medical teams; $80 \%$ of patients were HIV-infected (with a median CD4 count of $128 / \mathrm{mm}^{3}$ ). Approximately half of antiretroviral-naïve, HIV-infected patients started antiretroviral therapy in hospital. TB, predominantly extrapulmonary, was the most common diagnosis. Imported infections, notably severe falciparum malaria, accounted for a large number of the 81 different diagnoses in HIV-seronegative patients. Over half of all patients had co-morbidity complicating their clinical presentation. In-hospital mortality was $5.8 \%$, with overwhelming sepsis the cause in $40 \%$ of deaths, largely due to hospital-acquired infection, particularly in the HIV-infected cohort.

Conclusion. The overwhelming burden of ID in SA is revealed in this experience at GSH, a tertiary level referral hospital serving the Cape metropolitan area. The needs of the population warrant a reappraisal of human resource capacity and training in ID in SA.

S Afr Med J 2012;102(6):528-531.
The dual burden of human immunodeficiency virus (HIV) and tuberculosis (TB) dominates the health landscape of South Africa (SA), with approximately 5.5 million HIV-infected people and a TB incidence of about $1000 / 100000$ population. ${ }^{1}$ Up to $85 \%$ of new TB patients are co-infected with HIV, and together HIV and TB constitute the largest burden of premature mortality in SA, accounting for $22 \%$ of years of life lost. ${ }^{2}$ This overwhelming burden diverts attention from other infections that contribute significantly to mortality; lower respiratory tract infections, intestinal infectious diseases and septicaemia together accounted for $7.4 \%$ of years of life lost in 2009. ${ }^{2}$ Furthermore, poor infection control, a lack of proper antibiotic stewardship and irresponsible antibiotic prescribing in the public and private sectors has led to an increase in hospital-acquired infections (HAIs) and multidrug-resistant bacteria. ${ }^{3}$ HAIs increase morbidity, lengthen hospital stay (and costs), and impact negatively on in-hospital mortality. ${ }^{4}$ Imported infections also pose a significant threat to public health; the influenza pandemic of 2009 was a stark reminder of this potential threat, amid public panic and inability of an already overstretched health infrastructure to easily cope with the additional burden of infection.

Against this backdrop, infectious diseases (ID) became a recognised medical sub-specialty in SA in 2005. The challenge was to develop services at tertiary level hospitals that could support the primary and secondary level hospitals to cope with HIV-TB epidemics and

Division of Infectious Diseases and HIV Medicine, Department of Medicine, University of Cape Town

Mishal Pandie, MB ChB, Dip HIV

Helen van der Plas, MB ChB, FCP(SA), DTM\&H, Cert ID(SA)Phys

Gary Maartens, MB ChB, FCP(SA), DTM\&H, MMed

Marc Mendelson, BSc, MB BS, PhD, FRCP(UK), DTM\&H all other infections. ID units became responsible for training subspecialists, general physicians and paediatricians, allied healthcare workers, and medical students in ID. The challenges of providing specialist ID care and training are formidable, given the shortage of trained ID specialists and the lack of resources.

In April 2007, the Division of Infectious Diseases and HIV Medicine was established as a division of the Department of Medicine at Groote Schuur Hospital (GSH). In this article we describe the work of the ID unit and discuss the need to refocus SA's priorities towards training of physicians and allocation of resources to ID, in line with the burden of disease.

\section{Methods}

The GSH ID unit runs an inpatient consulting system, including out-of-hours emergency consultations, whereby referred patients are seen on a same-day basis by a junior member of the team and then by the consultant on call. Patients are co-managed with their primary medical team and are followed up daily until discharge or resolution of the infection episode. A prospective patient database was started in January 2008 to record the following clinical information: sex, referring team, HIV status (CD4 T-cell count, antiretroviral therapy (ART) on admission, ART during hospitalisation (including starts and changes), presenting clinical features, results of follow-up, final discharge diagnosis, presence of co-morbidity, death, and cause of death where known. The study was approved by the Human Research and Ethics Committee of the University of Cape Town.

A database was constructed to capture patient consultations. The database was checked weekly for inaccuracies by a consultant. Telephone consultations were not recorded. The discharge diagnosis and any co-morbidity data were captured. Statistical analysis was performed using STATA-10 software.

\section{Results}

The ID service at GSH was consulted by 2142 patients over the 4 -year period of 2008 - 2011, averaging 535 patients per year. Almost twothirds of referrals were from the Department of Medicine, with 37 
- $42 \%$ of referrals from secondary level medical teams and $15-22 \%$ from tertiary level medical sub-specialties. Dermatology accounted for $56 \%$ of all referrals over the 4 years, more than twice those from neurology, the second highest referring sub-specialty. In contrast to the predominance of general medical referrals, two-thirds of referrals from the Department of Surgery came from surgical sub-specialties such as vascular surgery and ENT departments, and only one-third from secondary level General Surgery. Together, the Departments of Obstetrics, Psychiatry and the Emergency and Intensive Care Units contributed $20 \%$ of all referrals.

\section{HIV-infected patients}

Approximately $80 \%$ of patients were HIV-infected and had markedly low CD4 counts (median 128/ $\mathrm{mm}^{3}$ ). The percentage of HIVinfected patients did not change significantly over time; however, the percentage of patients admitted to GSH on ART increased from 37.1\% to $48.1 \%$ between 2008 and 2011 . Notably, this was not associated with an increase in the median CD4 count on admission for those patients on ART. A total of $444 \mathrm{HIV}$-infected patients started ART while in hospital, typically as part of an accelerated programme for patients with very low CD4 counts $\left(<100 / \mathrm{mm}^{3}\right)$, followed by those with HIV-associated illnesses, the pathogenesis of which was related to HIV replication, e.g. HIV-related idiopathic thrombocytopenic purpura. ART was also commenced for untreatable opportunistic infections such as cryptosporidiosis.

$\mathrm{TB}$ was the discharge diagnosis in the majority of HIV-infected patients referred to the ID unit. In total, 665 of 1749 (38\%) HIVinfected patients had TB; this was the primary diagnosis in two-thirds of cases, and a co-morbid illness in the remaining third. Pulmonary TB was the commonest form of the disease, followed by disseminated, meningeal and abdominal TB. Multidrug-resistant TB was recorded in $17(4 \%)$ cases and a paradoxical immune reconstitution inflammatory

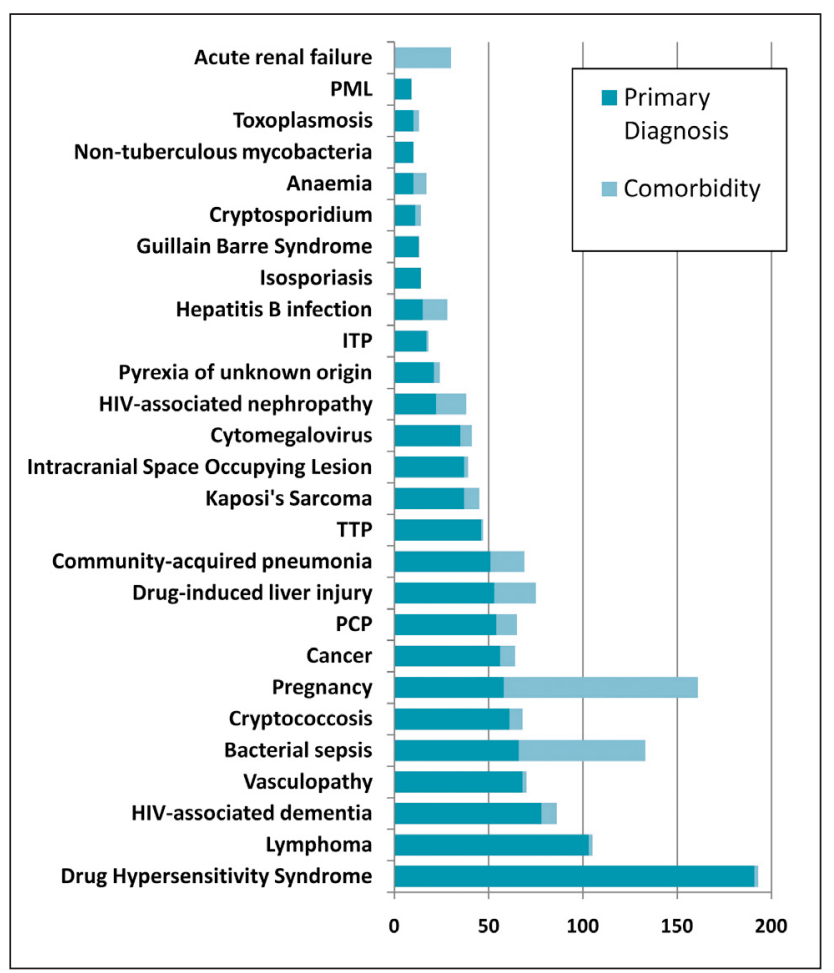

Fig. 1. Main primary diagnoses and co-morbid illnesses in HIV-infected patients, excluding tuberculosis. $P M L=$ progressive multifocal leuco-encephalopathy; $I T P=$ idiopathic thrombocytopenic purpura; $P C P=$ pneumocystis pneumonia; $T T P=$ thrombotic thrombocytopenic purpura.

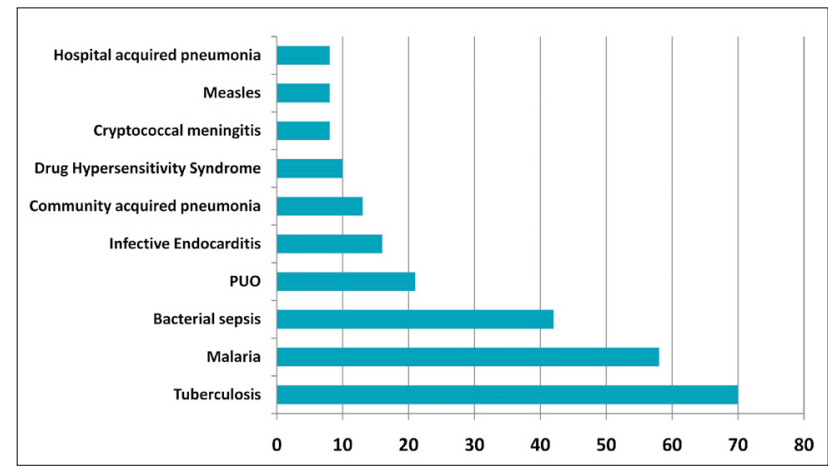

Fig. 2. Top 10 primary diagnoses in HIV-seronegative patients, 2008 - 2011.

syndrome (IRIS) in a further $4 \%$. Second after TB as a reason for consultation, were drug hypersensitivity syndromes (Stevens-Johnson syndrome, toxic epidermal necrolysis, drug reaction with eosinophilia and systemic symptoms) in 286 (16\%) patients (Fig. 1). Anti-TB drugs were implicated in 61 out of 286 (21\%) drug hypersensitivity syndrome cases, with non-nucleoside reverse transcriptase inhibitors and co-trimoxazole each implicated in a similar number. Cancer (lymphoma, Kaposi's sarcoma and non-AIDS-defining cancers) as primary diagnosis accounted for $11 \%$ of all referrals. Overall, $54 \%$ of patients had a co-morbid condition that was either directly linked to $\mathrm{HIV}$, such as opportunistic infection, or non-HIV-related illness such as acute renal failure, frequently the result of bacterial sepsis.

\section{HIV-seronegative patients}

The ID unit consulted on 385 HIV-seronegative patients, with 81 different diagnoses. TB was again the dominant primary diagnosis in $70(18 \%)$ patients, and was a co-morbid infection in a further 24 (Fig. 2). A total of 58 patients were admitted with severe Plasmodium falciparum malaria; 56 were men and 38 (65\%) were Somali immigrants. Half of all HIV-seronegative patients had a co-morbid condition such as diabetes, which compounded the seriousness of their primary illness. There were no significant differences between the referral sources of HIV-seronegative and HIV-infected patients, the majority of referrals originating from the Department of Medicine.

\section{Mortality}

Overall, there were 125/2 142 (5.8\%) deaths, 95/1 654 in the HIVinfected group and 30/281 in HIV-uninfected patients (with $p=0.002$ for the difference between the two groups). Overwhelming sepsis was the cause of death in 50 (40\%) patients; in 45 (36\%) the cause of death was uncertain. The remaining deaths were from end-stage TB, cancer, respiratory failure and severe falciparum malaria (3 cases). Postmortem examination was rarely performed.

\section{Hospital-acquired infection (HAI)}

There were 52 cases of HAI among HIV-infected patients, of whom $20(38 \%)$ died. Three of 12 (25\%) HIV-seronegative patients died as a result of HAI. The pathogen was not identified in $60 \%$ of cases of HAI resulting in death, typically due to omission of cultures prior to empirical prescribing of antibiotics. Pseudomonas aeruginosa, Acinetobacter baumannii and methicillin-resistant Staphylococcus aureus (MRSA) were isolated, with extended-spectrum $\beta$-lactamaseproducing Klebsiella pneumoniae the commonest bacterium isolated (in 5 cases).

\section{Discussion}

In the GSH ID unit $80 \%$ of the workload was HIV-related. The predominance of $\mathrm{TB}$ as the primary diagnosis in HIV-related 
consultations is in keeping with the massive burden of HIV-TB in the population. HIV-TB cases were predominantly extrapulmonary, affecting one or multiple (disseminated) sites. Nearly half of all HIVinfected patients not on ART, were adjudged to need to start ART in hospital, such was the advanced nature of their disease or the inherent role of HIV in the pathogenesis of their presenting illness. While in HIV-seronegative patients TB was again predominant, the role of the ID unit in managing very sick patients with falciparum malaria in a non-endemic province, attests to the importance of such units in guiding management of imported infections. HAIs were an important cause of in-hospital mortality, particularly in the HIVinfected cohort.

HIV-infected patients continue to be admitted to hospital with complications of advanced HIV infection in spite of significant advances in the number of patients starting ART at primary clinics since 2004. In Khayelitsha, Médecins sans Frontières (MSF) documented an increase in annual enrolment of patients to ART from 80 in 2001 to 2087 in 2007, ${ }^{5}$ with the median CD4 count of patients starting ART increasing from 43 to $162 / \mathrm{mm}^{3}$ between 2001 and $2010 .{ }^{6}$ Our hospitalised HIV patient cohort also showed an increase in the number admitted on ART from $37.1 \%$ to $48.1 \%$ over the 4-year period, although the median CD4 count remained static, reflecting perhaps the reduction in $\mathrm{CD} 4$ count that occurs in the setting of acute illness, or poor adherence with failure of ART. Forty-two per cent of hospitalised ART-naïve HIV-infected patients were started on ART in hospital, the commonest reason being a low CD4 count, which qualifies the patient for accelerated start under national guidelines. A significant proportion of patients starting ART in hospital were diagnosed with either AIDS-related or unrelated cancers, the rationale being to reduce the driving force of HIV in pathogenesis of AIDS-related cancers and/or to boost immunity, which might reduce recurrence or progression.

The dominance of TB as the primary diagnosis in HIV-positive and HIV-seronegative patients is not surprising, considering the massive burden of $\mathrm{TB}$ in the local population. The fact that over $60 \%$ of patients had extrapulmonary TB (EPTB) or disseminated TB reflects not only the increase in EPTB in HIV-infected patients in general, but the fact that EPTB more commonly presents a greater diagnostic challenge, requiring either a more experienced specialist opinion or diagnostic tests that are only available at a tertiary centre. Many of the HIV-TB cases presented as pyrexias of unknown origin, where baseline work-up had not revealed the diagnosis, or with drug resistance, TB-IRIS, or multiple opportunistic infections. The influence and burden of $\mathrm{TB}$ was also evident in patients presenting with drug hypersensitivity syndromes, one-fifth of which were related to anti-TB drugs.

The fact that over half of all patients had multiple co-morbidities, chief of which was bacterial sepsis, is a further indicator of the complexity of these cases. The most extreme example was an HIVinfected patient diagnosed with concurrent cryptococcal meningitis, pulmonary TB, Pneumocystis jirovecii pneumonia, chronic hepatitis B, oesophageal candidiasis and herpes genitalis.

The skewed distribution of HIV-infected patients may reflect a bias in referring practice. HIV is a relatively new infection in the daily practice of many physicians, who might not have been trained in management of HIV disease as medical students and junior doctors; their lack of confidence in treating HIV patients may lead to over-referral. In addition, the fact that GSH policy permitted only the ID team to initiate ART increased the referral of HIV-infected patients. Conversely, until recently, non-HIV-related ID was the sole realm of the general specialist, and hence an ID referral might not be considered necessary for many complex cases. However, because the majority of deaths from falciparum malaria in South Africa result from poor recognition and poor inpatient management, ${ }^{7}$ the 3 deaths that occurred in this cohort being no exception, such ID medical emergencies should preferably be co-managed by a trained ID sub-specialist. Our cohort of HIV-seronegative patients included 81 different diagnoses, many of which a general specialist would have little or no experience in managing, e.g. African trypanosomiasis, human fascioliasis, and botryomycosis.

While the burden of disease from HIV in SA is well documented, ${ }^{8}$ there have been no prospective studies detailing the contribution of ID units at tertiary institutions. Our study is the first in the country to document the range of patients seen by ID sub-specialists in a hospitalised cohort.

The limitations of this study include those inherent in all analyses of clinical databases over time, namely completeness and accuracy of data capture. Data were entered and checked by the same physicians and the discharge diagnoses entered by an experienced ID subspecialist. Our observations are limited to the inpatient clinical course and no comment on outcome after discharge is possible, as patients were not followed up after discharge.

The major limitation of this study is its inability to convey the work of our unit, other than formal inpatient consultations. We support other hospitals and physicians outside of GSH, via telephonic consultations, and advise on patients who do not require admission. We provide outreach and support to secondary level hospitals and to district level hospitals providing dedicated specialist TB services (in the form of ward rounds, outpatient clinics and teaching). As 1 of only 5 HPCSA-accredited ID training units in the country, we train ID sub-specialists, medical and family practitioner registrars, medical officers, students and allied healthcare practitioners in ID and HIV medicine. Positioned within our unit is the Cape Town GeoSentinel Travel Surveillance Site, which studies illness in travellers, who act as sentinels for new outbreaks and new infections, e.g. severe acute respiratory syndrome (SARS) in 2003.

The role that our unit plays in infection prevention and control (IPC) and antibiotic stewardship in the hospital is also not fully elucidated in this study. Like most institutions in the public sector, infection control is under-resourced and poorly practised. In general, prescribing habits of physicians are sub-standard and contribute to the rising incidence of multidrug-resistant bacterial infections. A recent pilot survey of antibiotic prescribing practice on medical wards at GSH revealed that an average of $62 \%$ of patients are prescribed one or more antibiotics, with $48 \%$ of prescriptions containing errors (M Mendelson - unpublished observations). These include a lack of indication for antibiotics, inappropriate duration of therapy, the use of multiple antibiotics with overlapping spectra, and incorrect dosing. ID trainees spend 6 months in the laboratory and 18 months on clinical service, during which they learn rational antibiotic prescribing and to lead antibiotic stewardship teams in tertiary and secondary level hospitals. Our team augments the work of the hospital's IPC sisters by training, advising wards on appropriate IPC, and participating in policy development.

Our findings generate a number of research questions that need to be addressed: among patients commencing ART as inpatients, what is their long-term outcome and does it differ from patients who are started on ART in outpatient clinics? (A recent study from the Eastern Cape showed that retention in care was reduced if pregnant women were started on ART as inpatients, ${ }^{9}$ perhaps because pregnant women view ART as primarily to protect their babies). Is HAI, as a cause of death in HIV-infected patients, peculiar to tertiary hospital inpatients or does it apply to all HIV-infected hospitalised patients? 
Human resource capacity building lags behind the national burden of ID. The complexity of patients described in this study, which is mirrored at secondary and district level hospitals, demands either an increase in the number of sub-specialists at tertiary level who can support the secondary and primary levels through outreach and support, or a national policy to appoint ID-trained subspecialists at secondary level hospitals to provide support also at the primary level. As capacity building will be a long-term project, a policy decision that all medical and family practitioner registrars rotate through a recognised ID unit as part of their training should be put into effect without delay.

Acknowledgements. HvdP and MP were funded by, and MM received financial support from, PEPFAR/USAID through the ANOVA Health Institute.

\section{References}

1. World Health Organization. Global Tuberculosis Control: WHO report 2011. Geneva: WHO, 2011.

2. Provincial Government of the Western Cape. Recommendations for Policy in the Western Cape Province for the prevention of major infectious diseases, including HIV/AIDS and tuberculosis, June 2007. http:// www.westerncape.gov.za/eng/pubs/reports_research/W/157844 (accessed 9 January 2012).

3. Shlaes DM, Gerding DN, John JF jun, et al. Society for Healthcare Epidemiology of America and Infectious Diseases Society of America Joint Committee on the Prevention of Antimicrobial Resistance: Guidelines for the prevention of antimicrobial resistance in hospitals. Clin Infect Dis 1997;25:584-599. 4. Hassan M, Tuckman HP, Patrick RH, Kountz DS, Kohn JL. Cost of hospital-acquired infection. Hospital Topics 2010;88(3):82-89.

5. Boulle A, van Cutsem G, Hildebrand K, et al. Seven-year experience of a primary care antiretroviral treatment programme in Khayelitsha, South Africa AIDS 2010:24: 563-572.

6. Médecins sans Frontières. Khayelitsha 2001-2011. Activity report: 10 years of HIV/TB care at primary health care level. http://www.health-e.org.za/documents/6eadc65d79595b1a2cb0c86337161a4c.pdf (accessed 17 January 2012).

7. Mehta U, Durrheim DN, Blumberg L, et al. Malaria deaths as sentinel events to monitor healthcare delivery and antimalarial druo safety. Trop Med Int Health 2007:12(5):617-628.

8. Joint United Nations Programme on HIV/AIDS (UNAIDS). Global Report: UNAIDS report on the global AIDS epidemic 2010. http://www.unaids.org/globalreport/documents/20101123_ GlobalReport_full_en.pdf (accessed 20 January 2012).

9. Boyles T, Wilkinson LS, Leisegang R, Maartens G. Factors influencing retention in care after starting antiretroviral therapy in rural South Africa. PLoS One 2011;6(5);e19201
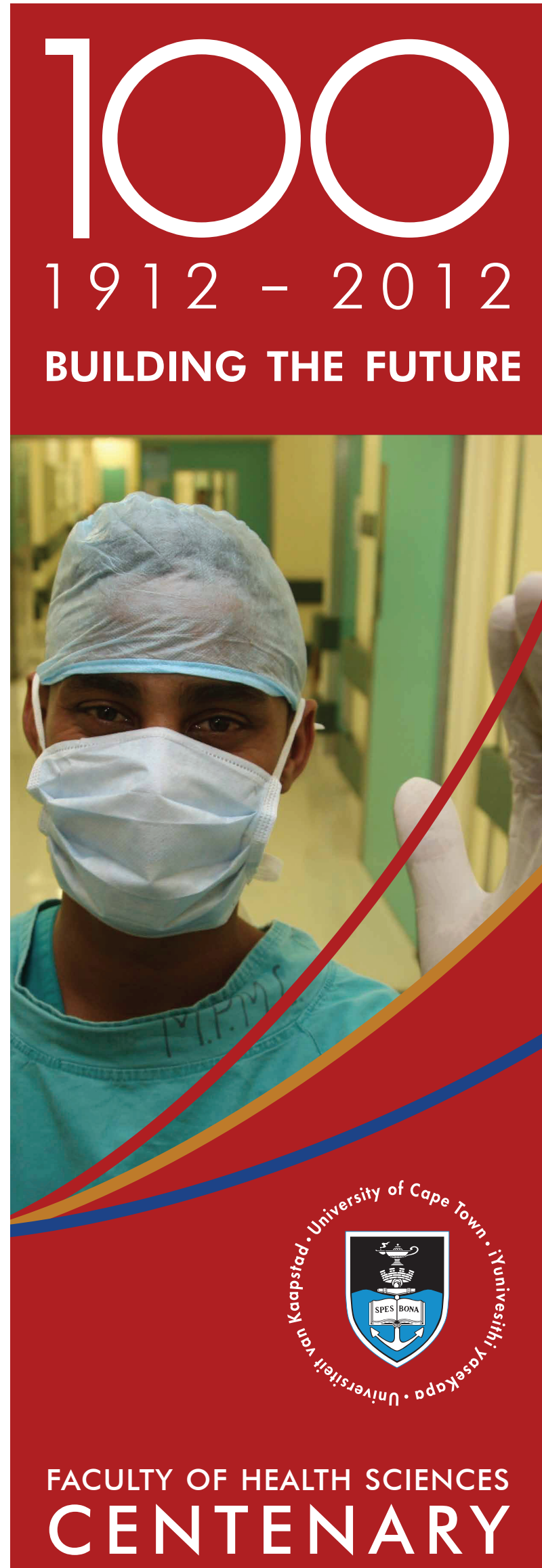